\title{
Article \\ On transcendental numbers: new results and a little history
}

\author{
Solomon Marcus ${ }^{1}$ and Florin F. Nichita ${ }^{1, *}$ \\ ${ }^{1}$ Institute of Mathematics of the Romanian Academy, 21 Calea Grivitei Street, 010702 Bucharest, Romania \\ * E-Mail: Florin.Nichita@imar.ro; Tel: (+40) (0) 2131965 06; Fax: (+40) (0) 213196505
}

\begin{abstract}
Bringing toghether mathematical and philosophical ideas related to transcendental numbers, this paper begins with a survey on transcendence and transcendental numbers, it then presents several properties of the transcendental numbers $e$ and $\pi$, and then it gives the proof of a new inequality for transcendental numbers. Also, in relationship with these topics, we study solutions to the Yang-Baxter equation from hyperbolic functions and from logical implication.
\end{abstract}

Keywords: Euler's relation, transcendental numbers; transcendental operations / functions; transcendence; Yang-Baxter equation, hyperbolic functions, logical implication

MSC: 01A05; 11D09; 11T23; 33B10; 16T25

\section{Introduction}

One of the most famous formulas in mathematics, the Euler's relation:

$$
e^{\pi i}+1=0
$$

contains the transcendental numbers $e$ and $\pi$, the imaginary number $i$, the constants 0 and 1 , and (transcendental) operations. Beautiful, powerful and surprising, it has changed the mathematics forever.

Another interesting relation is the following (see [1]):

$$
\left|e^{i}-\pi\right|>e,
$$

and it has an interesting geometrical interpretation (see Fig. 1).

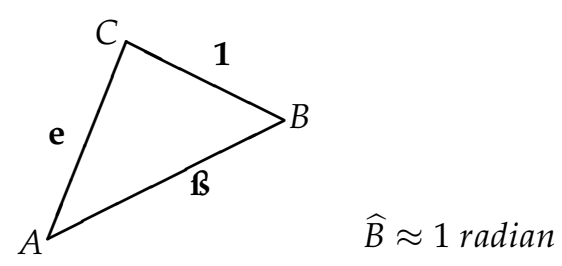

Figure 1. An interpretation of the relation $\left|e^{i}-\pi\right| \approx e$.

In some sense, the formulas (1) and (2) can be unified, and we will explain this in Section 3.

The next section of the current paper is a survey on transcendental numbers, transcendental functions, transcendental operations and transcendence (following the ideas from [2,3]). Section 3 deals with identities and inequalities of transcendental numbers, and the proof of formula (2). In Section 4 we start with some comments, and then we propose some open problems. Section 5 contains two important constructions: solutions to the Yang-Baxter equation from hyperbolic functions and from material implication. The last section is about conclusions. 


\section{Transcendence and transcendental numbers in mathematics}

The term "transcendence" has been introduced to express issues related to the Divinity. Implying initially the aforementioned attributes, it has produces an inflation of uses nowadays: movies, novels, jazz albums, corporations, game brands, poems, internet websites, internet blogs, names of all kinds of doctrines, philosophies, etc. The "trans" prefix comes from Latin, implying passing through a certain area, and it is all around: translation, transport, transmission, transformation, transplant, transparent, transgress, transdisciplinarity etc.

One talked for the first time about transcendence in mathematics in the 18th century, and it was Leonhard Euler who initiated this discussion (see [4]). Leibniz was the first referring to transcendence in mathematics (see [5]). Euler does not refer directly to transcendental numbers (as it happened, subsequently, in the 19th century), but he refers to transcendental operations. The non-transcendental operations are: the addition, the subtraction, the multiplication, the division, the exponentiation and the rooting (applied to integers and to those numbers that are obtained from them by such operations). Why all these operations are considered to be non-transcendent? They are considered to be algebraic operations, because all numbers generated in this way are roots of some algebraic equations; we refer to those equations expressed by polynomial structures, where all mentioned operations are applied for finite number of times (focus on finite) and, as soon as this is not the case, we enter in the field of transcendence. Therefore, we are suddenly suggested that, in mathematics, the idea of transcendence is essentially related to the idea of infinity. But not to any kind of infinity. For Euler, the entry in mathematical transcendence is in the differential and integral calculus. Why is Euler considering the exponentiation a to $x$, the logarithm, the trigonometric sinus-cosine functions as being all transcendental functions and operations? Because they are immediately associated, in a way or another, to the mathematical idea of integral, they are immediately associated to expressions including an integral (for instance, the $\operatorname{logarithm}$ is associated to the integral of $\mathrm{dx} / \mathrm{x})$. For Euler, the function $\log (\mathrm{x})+7$ is transcendent, but $\log (7)+x$ is algebraic.

The passing from non-transcendent to transcendent is gradual. Such an assertion deviates us from mathematical rigor, but it consolidates our understanding. We have in mathematics the situation of irrational numbers, where the word irrational would suggest transcendence, but it is immediately completed by the word algebraic the negation of transcendent, such as the square root of 2 . We may consider the algebraic irrationals as a passing bridge, therefore intermediary when passing from non-transcendent to transcendent. It is not rigorous, but it is suggestive. We must add another thing: we talk about rational numbers (such as 2/ 3 or -3/7) and irrational numbers, and we are tempted to associate such irrational numbers as square root of 2 with something beyond reason. But the Latin etymology clarifies the fact that rational is sending here to ratio as fraction; so, in modern understanding, as a number of the form $a / b$, where $a$ and $b$ are integers, with $b$ different from zero.

Joseph Liouville is the first who managed to encounter an example of transcendental numbers. This important discovery happened in the year 1844. Liouville introduced a class of real numbers wearing subsequently his name. A real number $x$ is a Liouville number if there exist an integer $b$ higher or equal to 2 and an infinite sequence of integers $\left(a_{1}, a_{2}, . ., a_{n}, \ldots\right)$ so that $\mathrm{x}$ is the sum of the series having as general term the ratio between $a_{k}$ and the power of exponent $\mathrm{k}$ ! of $\mathrm{b}$. (For $\mathrm{b}=10$ and $a_{k}=1$ for any $\mathrm{k}$, x becomes Liouville's constant.) Concerning his numbers (see also Remark 4.4), Liouville proves that they are not algebraic; they are transcendental. It was the first example of non-algebraic real numbers. Another presentation of Liouville's numbers stands on the manner of approximation by rational numbers: $x$ is a Liouville number if for any natural number $n$ there are integers $p$ and $q$ with $q$ higher or equal to 2 , such that the absolute value of the difference between $x$ and $p / q$ is strictly between zero and the fraction having 1 at the nominator and the value of $q$ power $n$, at the denominator. It is thus clear that Liouville's numbers have the privilege of a tight approximation by rational numbers. This fact is against our intuitive expectations, because it shows that in some respects transcendental numbers are nearer to rational numbers than algebraic irrationals. 
The manner how the rational numbers are diligently "running" to get close to the transcendent numbers urges us to see the transcendence process in exactly the infinite range of approximations of transcendental numbers. This situation suggests us to generally regard transcendence as result of an asymptotic process when all stages are in the terrestrial universe, but the infinity of the number of stages makes impossible to be crossed in real time. Such a view increases the rightfulness of the hypothesis of absence of a sharp border between transcendent and terrestrial. It is noticed that the Liouville numbers are encountered in remarkable classes of real numbers, but as rare exceptional phenomenon.

There are other philosophical enigmas related to mathematical transcendence. One would expect that transcendental numbers, such as $\pi$ or $e$, to be somehow farther from rational numbers, than irrational algebraic numbers. But in terms of approximation by rational numbers, it seems that this does not happen. It must be noticed, as well, that transcendental numbers are more than algebraic numbers, but "more" means here a metaphoric extension of such qualification, from finite to infinite. The mathematicians are expressing this by the following statement: "The algebraic numbers form a countable set, while the transcendental numbers form an uncountable set; it is a set of the power of the continuum".

\section{Transcendental numbers: identities and inequalities}

The following identities which contain the transcendental numbers $e$ and $\pi$ are well-known:

$$
\begin{gathered}
\int_{-\infty}^{+\infty} e^{-x^{2}} d x=\sqrt{\pi} \\
\int_{-\infty}^{+\infty} e^{-i x^{2}} d x=\sqrt{\frac{\pi}{2}}(1-i) .
\end{gathered}
$$

These formulas, unified in the paper [6], can be proved by contour integration.

Other inequalities for $e$ and $\pi$ (from [1]) are quite new; for example, we list just two of them:

$$
\begin{aligned}
& \left|e^{1-z}+e^{\bar{z}}\right|>\pi \quad \forall z \in \mathbb{C}, \\
& \int_{a}^{b} e^{-x^{2}} d x<\frac{e^{e}}{\pi}\left(\frac{1}{e^{\pi a}}-\frac{1}{e^{\pi b}}\right) .
\end{aligned}
$$

Formula (2) will be proved by hand below.

The inequality $\left|e^{i}-\pi\right|>e$ is equivalent to $(\pi+e)(\pi-e)+1>2 \pi \cos 1$. Now, we use the estimations $\pi>3.141, e<2.719,(\pi+e)(\pi-e)+1>3.47292$ and $\cos 1<1-\frac{1}{2}+\frac{1}{4 !}=\frac{13}{24}$, $2 \pi \cos 1<3.142 \times \frac{13}{12}=3.4038(3)$. (This proof was improved by Dr. Cezar Joita.)

Remark 3.1. Let us consider the two variable complex function $f: \mathbb{C} \times \mathbb{C} \rightarrow \mathbb{R}, f(z, w)=\left|e^{z}+e^{w}\right|$. The formulas (1), (2) and (5) can be unified using the function $f(z, w)$ :

$$
f(\pi i, 0)=0, \quad f(1-z, \bar{z})>\pi \forall z \in \mathbb{C}, \quad f(i, \pi i+\ln \pi)>e .
$$

\section{Further comments and open problems}

Remark 4.1. Related to our Section 2, one could consider the following equation

$$
x^{2}-\pi x+\left(1+\frac{1}{r}\right)^{r}=0, \quad r \in \mathbb{Q}^{*},
$$

which has real solutions for $r$ sufficiently small, but the "limit" equation $x^{2}-\pi x+e=0$, has no real solutions because $\Delta=\pi^{2}-4 e<0$.

The question if $\Delta=\pi^{2}-4 e$ is a transcendental number is an open problem. 
Figure 2. Some Jordan curves. A circle with diameter $\pi$ and a square with edges of length $e$ have almost the same perimeter.

Remark 4.2. Resembling the problem of squaring the circle, the geometrical interpretation of the formula $\pi^{2}<4 e$ could be stated as: "The length of the circle with diameter $\pi$ is almost equal (and less) to the perimeter of a square with edges of length $e^{\prime \prime}$ (see the Figure 2). In this case, the area of the above circle is greater than the area of the above square, because $\pi^{3}>4 e^{2}$.

Remark 4.3. We now propose some open problems related to $\pi$.

For a Jordan curve in the Euclidean plane (i.e., an arbitrary convex closed curve-see Fig. 2), we consider the smallest diameter $(d)$ and the maximum diameter $(D)$. (These can be found by considering the center of mass of a body which corresponds to the domain inside the given curve.)

(i) If $L$ is the length of the given curve then:

$$
\frac{L}{D} \leq \pi \leq \frac{L}{d}
$$

(ii) Moreover, the first inequality becomes equality if and only if the second inequality becomes equality if and only if the given curve is a circle.

(iii) If the area of the domain inside the given curve is $A$, then $d D>A$.

(iv) The equation $x^{2}-\frac{L}{2} x+A=0$ is not completely solved; for example, if the given curve is an ellipse, solving this equation is an unsolved problem.

Remark 4.4. Solving the equation $x^{2}+\sum_{0}^{\infty} 10^{-k !} x+\sum_{1}^{\infty} 10^{-k !}=0$, in which two coefficients are Liouville numbers, by using the quadratic formula is hard; however, one can use the formula $A x^{2}+(A+C) x+$ $C=(A x+C)(x+1)$ in order to solve this equation. The solution $x=-1$ could be observed directly.

\section{Transcendental numbers in mathematical physics}

In some special issues on Hopf algebras, quantum groups and Yang-Baxter equations, several papers [7-22], as well the feature paper [23], covered many topics related to the Yang-Baxter equation, ranging from mathematical physics to Hopf algebras, from Azumaya Monads to quantum computing, and from Mathematical Logic to Rota-Baxter equations.

The terminology of this section is compatible with the above cited papers. $V$ is a complex vector space, and $I_{j}: V^{\otimes j} \rightarrow V^{\otimes j} \quad \forall j \in\{1,2\}$ are identity maps. We consider $J: V^{\otimes 2} \rightarrow V^{\otimes 2}$ a linear map which satisfies $J \circ J=-I_{2}$ and $J^{12} \circ J^{23}=J^{23} \circ J^{12}$, where $J^{12}=J \otimes I_{1}, J^{23}=I_{1} \otimes J$.

Then, $R(x)=\cos x I_{2}+\sin x J$ satisfies the colored Yang-Baxter equation:

$$
R^{12}(x) \circ R^{23}(x+y) \circ R^{12}(y)=R^{23}(y) \circ R^{12}(x+y) \circ R^{23}(x) .
$$

The proof of (6) could be done by writing $R(x)=e^{x J}$, and checking that (6) reduces to 
$x J^{12}+(x+y) J^{23}+y J^{12}=y J^{23}+(x+y) J^{12}+x J^{23}$.

Based on results from the previous section, a related formula to the relation

$$
e^{\pi J}+I_{4}=0_{4} \quad J, I_{4}, 0_{4} \in \mathcal{M}_{4}(\mathbb{C})
$$

is the following inequality (which we leave without proof):

$$
\left.X^{2}+e I_{2}>\pi X \quad \forall X \in \mathcal{M}_{2}\left(\mathbb{R}_{+}^{*}\right)\right), \operatorname{trace}(X)>\pi .
$$

A generalization, with $J \circ J=p I_{2}, J^{12} \circ J^{23}=q J^{23} \circ J^{12}, p q=-1$, was studies in [24].

Replacing just the condition $J^{12} \circ J^{23}=J^{23} \circ J^{12}$ with $J^{12} \circ J^{23}=-J^{23} \circ J^{12}$, the authors of [25] obtained interesting results (a new realization of doubling degeneracy based on emergent Majorana operator, new solutions for the Yang-Baxter equation, etc).

With this cases we enter into the world of the quaternions and Clifford algebras. We present another situation below.

Theorem 5.1. (Solutions to the Yang-Baxter equation from hyperbolic functions)

Let $J: V^{\otimes 2} \rightarrow V^{\otimes 2}$ a linear map which satisfies $J \circ J=I_{2}$ and $J^{12} \circ J^{23}=J^{23} \circ J^{12}$.

Then, $R(x)=\cosh x I_{2}+\sinh x$ J satisfies the colored Yang-Baxter equation (6).

Proof. The equality $\left(\cosh x+\sinh x J^{12}\right)\left(\cosh x+\sinh x J^{23}\right)\left(\cosh x+\sinh x J^{12}\right)=(\cosh x+$ $\left.\sinh x J^{23}\right)\left(\cosh x+\sinh x J^{12}\right)\left(\cosh x+\sinh x J^{23}\right)$ follows from some properties of hyperbolic functions, $\cosh (x+y)=\cosh x \cosh y+\sinh x \sinh y \quad$ and $\sinh (x+y)=\sinh x \cosh y-\cosh x \sinh y$, and from $J \circ J=I_{2}$ and $J^{12} \circ J^{23}=J^{23} \circ J^{12}$.

Remark 5.2. For example, in dimension two, the matrix form of the above operator J could be:

$$
\left(\begin{array}{llll}
0 & 0 & 0 & 1 \\
0 & 0 & 1 & 0 \\
0 & 1 & 0 & 0 \\
1 & 0 & 0 & 0
\end{array}\right)
$$

The corresponding matrix for $R(x)$ in this case can be expresses as follows:

$$
\left(\begin{array}{cccc}
e^{x}+e^{-x} & 0 & 0 & e^{x}-e^{-x} \\
0 & e^{x}+e^{-x} & e^{x}-e^{-x} & 0 \\
0 & e^{x}-e^{-x} & e^{x}+e^{-x} & 0 \\
e^{x}-e^{-x} & 0 & 0 & e^{x}+e^{-x}
\end{array}\right)
$$

Theorem 5.3. (Solutions to the Yang-Baxter equation from the material implication)

For a Boolean algebra, the map $R(a, b)=(a \rightarrow b, a)$ is a solution for the constant Yang-Baxter equation:

$$
R^{12} \circ R^{23} \circ R^{12}=R^{23} \circ R^{12} \circ R^{23} .
$$

Proof. The equality $R^{12} \circ R^{23} \circ R^{12}(a, b, c)=R^{23} \circ R^{12} \circ R^{23}(a, b, c)$ implies $a \rightarrow(b \rightarrow c)=$ $(a \rightarrow b) \rightarrow(a \rightarrow c)$, but $a \rightarrow(b \rightarrow c)=\bar{a} \vee(\bar{b} \vee c)=(\bar{a} \vee \bar{b} \vee c) \wedge(\bar{a} \vee a \vee c)=(a \wedge \bar{b}) \vee(\bar{a} \vee c)=$ $(a \rightarrow b) \rightarrow(a \rightarrow c)$.

\section{Conclusions}

Our main results are the Theorems 5.1 and 5.2, the proof by hand of the formula (2), and the unification of the formulas (1) and (2). Several open problem are presented (Remarks 4.1 and 4.3). We 
used results and concepts from Algebra, Mathematical Analysis, Mathematical Physics, Geometry, History of Mathematics, Numerical Analysis, Epistemology, Philosophy etc. Because there is a huge number of new disciplines, it is important to have a transdisciplinary understanding of the world: a transdisciplinary approach (see [27-30]) attempts to discover what is between disciplines, across different disciplines, and beyond all disciplines. Transcendence is a concept which plays an important role in theology, in science and in art; it can be considered beyond all disciplines. The Yang-Baxter equation appears across different disciplines. Mathematical Physics is at the border of two disciplines.

The author of [26] considers two types of scientists: birds (they resemble scientists with a broad vision, who try to unify theories, who obtain results of interest for a large readership) and frogs (which are less influential). Solomon Marcus used the terms of Francis Bacon (Novum Organum), bees versus ants in this matter, and he also refers to spiders (scientists building large networks of results).

We conclude with some historical facts. Euler was born in Basel (on 15 April 1707), and he received his Master of Philosophy from University of Basel. On 17 March 2016, Solomon Marcus has pased away.

Acknowledgements. We would like to thank all those who helped us to improve our initial preprint.

1. Nichita, F.F. On Transcendental Numbers, Axioms 2014, 3(1), 64-69.

2. Marcus, S. Transcendence, as a universal paradigm (in Romanian), Convorbiri Literare 2014, February, 17-28.

3. Marcus, S. Transcendence, as a universal paradigm, BALANCE, A Club of Rome Magazine 2015, no.1, March, 50-70.

4. Petrie, B.J. Leonhard Euler's use and understanding of mathematical transcendence, Historia Mathematica 2012, 39, 280-291.

5. Breger, H. Leibniz Einfuhrung des Transzendenten, 300 Jahre „Nova Methodus” von G.W. Leibniz (1684-1984). Studia Leibnitiana 1986, Sonderheft XIV, 119-132.

6. Desbrow, D. On Evaluating $\int_{-\infty}^{+\infty} e^{a x(x-2 b)} d x$ by Contour Integration Round a Parallelogram, The Amer. Math. Month. 1998, Vol 105, Number 8, 726-731.

7. Kanakoglou, K. Gradings, Braidings, Representations, Paraparticles: Some Open Problems, Axioms 2012, 1(1), 74-98.

8. Underwood R.G. Quasi-triangular Structure of Myhill-Nerode Bialgebras, Axioms. 2012, 1(2), 155-172.

9. Schmidt, J.R. From Coalgebra to Bialgebra for the Six-Vertex Model: The Star-Triangle Relation as a Necessary Condition for Commuting Transfer Matrices. Axioms 2012, 1(2), 186-200.

10. Nichita, F.F.; Zielinski, B. The Duality between Corings and Ring Extensions. Axioms 2012, 1(2), 173-185.

11. Links, J. Hopf Algebra Symmetries of an Integrable Hamiltonian for Anionic Pairing, Axioms 2012, 1(2), $226-237$.

12. Hoffnung, A.E. The Hecke Bicategory, Axioms 2012, 1(3), 291-323.

13. Nichita, F.F. Yang-Baxter systems, algebra factorizations and braided categories, Axioms 2013, 2(3), 437-442.

14. Lebed, V. R-Matrices, Yetter-Drinfel'd Modules and Yang-Baxter Equation, Axioms 2013, 2(3), 443-476.

15. Iordanescu, R.; Nichita, F.F.; Nichita I.M. The Yang-Baxter Equation, (Quantum) Computers and Unifying Theories, Axioms 2014, 3(4), 360-368.

16. Mesablishvili, B.; Wisbauer, R. Azumaya Monads and Comonads, Axioms 2015, 4(1), 32-70.

17. Saramago, R.M. Generalized Yang-Baxter Operators for Dieudonné Modules. Axioms 2015, 4, 177-193.

18. Nichita, F.F. Yang-Baxter Equations, Computational Methods and Applications. Axioms 2015, 4, 423-435.

19. Smith, J. Quantum Quasigroups and the Quantum Yang-Baxter Equation. Axioms 2016, 5, 25.

20. Oner, T.; Senturk, I.; Oner, G. An Independent Set of Axioms of MV-Algebras and Solutions of the Set-Theoretical Yang-Baxter Equation. Axioms 2017, 6, 17.

21. Lentner, S.; Lochmann, A. Factorization of Graded Traces on Nichols Algebras. Axioms 2017, 6, 32.

22. Gubarev, V. Universal Enveloping Commutative Rota-Baxter Algebras of Pre- and Post-Commutative Algebras. Axioms 2017, 6, 33.

23. Nichita, F.F. Introduction to the Yang-Baxter Equation with Open Problems, Axioms 2012, 1(1), $33-37$.

24. Nichita, F.F. Jordan algebras, Jordan coalgebras and unification theories, Editions Universitaires Europeennes (2017), ISBN: 978-3-639-62347-5. 
25. Yu, L.W.; Ge, M.L. More about the doubling degeneracy operators associated with Majorana fermions and Yang-Baxter equation, Scientific Reports 2015, 5, Article number: 8102, 7 pages.

26. Dyson, F. Birds and Frogs, Notices of the AMS 2009, Vol. 56, No. 2, 212-223.

27. Nicolescu, B. Manifesto of Transdisciplinarity, State University of New York (SUNY) Press, New York, translation in English by Karen-Claire Voss, 2002.

28. Nicolescu, B. Transdisciplinarity - past, present and future, in Moving Worldviews - Reshaping sciences, policies and practices for endogenous sustainable development 2006, COMPAS Editions, Holland, edited by Bertus Haverkort and Coen Reijntjes, 142-166.

29. Nicolescu, B. The unexplected way to holiness: Simone Weil (II), (in Romanian), Convorbiri Literare 2017, October, No. 10(262),26-28.

30. Nichita, F.F. On Models for Transdisciplinarity, Transdisciplinary Journal of Engineering and Science 2011, Vol. 2011, 42-46. 